\title{
Variational Integrators for Maxwell's Equations with Sources
}

\author{
A. Stern ${ }^{1}$, Y. Tong ${ }^{1,2}$, M. Desbrun ${ }^{1}$, and J. E. Marsden ${ }^{1}$ \\ ${ }^{1}$ California Institute of Technology, USA \\ ${ }^{2}$ Michigan State University, USA
}

\begin{abstract}
In recent years, two important techniques for geometric numerical discretization have been developed. In computational electromagnetics, spatial discretization has been improved by the use of mixed finite elements and discrete differential forms. Simultaneously, the dynamical systems and mechanics communities have developed structure-preserving time integrators, notably variational integrators that are constructed from a Lagrangian action principle. Here, we discuss how to combine these two frameworks to develop variational spacetime integrators for Maxwell's equations. Extending our previous work, which first introduced this variational perspective for Maxwell's equations without sources, we also show here how to incorporate free sources of charge and current.
\end{abstract}

\section{INTRODUCTION}

In computational electromagnetics, as in an increasing number of other fields in applied science and engineering, there is both practical and theoretical interest in developing geometric numerical integrators. These numerical methods preserve, by construction, various geometric properties and invariants of the continuous physical systems that they approximate. This is particularly important for applications where even high-order methods may fail to capture important features of the underlying dynamics. In this short paper, we show that the traditional Yee scheme and extensions can be derived from the Euler-Lagrange equations of a discrete action, i.e., by designing an electromagnetic variational integrator, including free sources of charge and current in non-dissipative media. Furthermore, we present how to use this discrete geometric framework to allow for asynchronous time stepping on unstructured grids, as recently introduced in Stern et al. [10].

Variational integrators (not to be confused with variational methods such as finite element schemes) were originally developed for geometric time integration, particularly to simulate dynamical systems in Lagrangian mechanics. The key idea is the following: rather than approximating the equations of motion directly, one discretizes the Lagrangian and its associated action integral (e.g., using a numerical quadrature rule), and then derives a structure-preserving approximation to the equations of motion by applying Hamilton's principle of stationary action. Since the numerical method is derived from a Lagrangian variational principle, some important results from Lagrangian dynamics carry over to the discretized system, including Noether's theorem relating symmetries to conserved momentum maps, as well as the fact that the Euler-Lagrange flow is a symplectic mapping. (See Marsden and West [8], Lew et al. [7].)

Overview. To develop a variational integrator for Maxwell's equations, the discrete Hamilton's principle needs to incorporate more than just the time discretization, as in mechanics; spatial discretization also needs to be handled carefully. Building upon mixed finite elements in space $[2,5,9]$, we treat the electromagnetic Lagrangian density as a discrete differential 4-form in spacetime. Extremizing the integral of this Lagrangian density with fixed boundary conditions directly leads to discrete update rules for the electromagnetic fields, with either uniform or asynchronous time steps across the various spatial elements.

\section{REVIEW OF MAXWELL'S EQUATIONS IN SPACETIME}

Electromagnetic Forms. Let $A$ be a 1-form on spacetime, called the electromagnetic potential, and then define the Faraday 2 -form to be its exterior derivative $F=d A$. Given a time coordinate $t$, this splits into the components

$$
F=E \wedge d t+B
$$

where $E$ is the electric displacement 1-form and $B$ is the magnetic flux 2-form, both defined on the spacelike Cauchy surfaces $\Sigma$ with constant $t$. If $*$ is the Hodge star associated to the spacetime metric, then we can also split the dual 2 -form

$$
* F=\left(*_{\mu} B\right) \wedge d t-*_{\epsilon} E=H \wedge d t-D
$$


where (again, restricted to Cauchy surfaces) $H$ is the magnetic displacement 1 -form, $D$ is the electric flux 2 -form, and $*_{\mu}$ and $*_{\epsilon}$ are respectively the magnetic permeability and electric permittivity. Finally, for systems with free sources, there is a source 3-form $\mathcal{J}$, satisfying the continuity of charge condition $d \mathcal{J}=0$. In terms of coordinates, this can be split into

$$
\mathcal{J}=J \wedge d t-\rho,
$$

where $J$ is the current density 2 -form and $\rho$ is the charge density 3 -form on Cauchy surfaces.

Maxwell's Equations. With the spacetime forms and operators defined above, Maxwell's equations become

$$
d F=0, \quad d * F=\mathcal{J} .
$$

Note that the first equation follows automatically from $F=d A$, since taking the exterior derivative of both sides yields $d F=d d A=0$. The second equation is consistent with the continuity of charge condition, since $d \mathcal{J}=d d * F=0$.

Lagrangian Formulation. Given the electromagnetic potential 1-form $A$ and source 3 -form $\mathcal{J}$, we can define the Lagrangian density to be the 4-form

$$
\mathcal{L}=-\frac{1}{2} d A \wedge * d A+A \wedge \mathcal{J}
$$

with the associated action functional $S[A]=\int_{X} \mathcal{L}$ taken over the spacetime domain $X$. Suppose that $\alpha$ is a variation of $A$, vanishing on the boundary $\partial X$. Varying the action along $\alpha$ yields

$$
\mathbf{d} S[A] \cdot \alpha=\int_{X}(-d \alpha \wedge * d A+\alpha \wedge \mathcal{J})=\int_{X} \alpha \wedge(-d * d A+\mathcal{J}) .
$$

Hamilton's principle of stationary action states that this variation must equal zero for any such $\alpha$, implying the Euler-Lagrange equations $d * d A=\mathcal{J}$. Finally, substituting $F=d A$ and recalling that $d F=d d A=0$, we see that this is equivalent to Maxwell's equations.

\section{GEOMETRIC PROPERTIES OF MAXWELL'S EQUATIONS}

As written in terms of $F$ above, Maxwell's equations have 8 components: 6 dynamical equations, which describe how the fields change in time, and 2 "divergence constraints" containing only spatial derivatives. The fact that these constraints are automatically preserved by the dynamical equations (and can therefore effectively be ignored except at the initial time) comes directly from the differential gauge symmetry and Lagrangian variational structure. We discuss these geometric properties here, with a view towards developing numerical methods that preserve them.

Reduction by Gauge Fixing. Maxwell's equations are invariant under gauge transformations $A \mapsto A+d f$ for any scalar function $f$, since taking the exterior derivative maps $F \mapsto F+d d f=F$. Therefore, given a time coordinate $t$, we can fix the gauge so that $A \cdot \frac{\partial}{\partial t}=0$, i.e., $A$ has only spacelike components. This partial gauge fixing is known as the Weyl gauge. Restricted to this subspace of potentials, the Lagrangian then becomes

$$
\begin{aligned}
\mathcal{L} & =-\frac{1}{2}\left(d_{t} A+d_{\Sigma} A\right) \wedge *\left(d_{t} A+d_{\Sigma} A\right)+A \wedge \mathcal{J} \\
& =-\frac{1}{2}\left(d_{t} A \wedge * d_{t} A+d_{\Sigma} A \wedge * d_{\Sigma} A\right)+A \wedge J \wedge d t
\end{aligned}
$$

Here, we have adopted the notation $d_{t}$ and $d_{\Sigma}$ for the exterior derivative taken only in time and in space, respectively; in particular, we then have $d_{t} A=E \wedge d t$ and $d_{\Sigma} A=B$.

Next, varying the action along a restricted variation $\alpha$ that vanishes on $\partial X$,

$$
\begin{aligned}
\mathbf{d} S[A] \cdot \alpha & =\int_{X}\left(d_{t} \alpha \wedge D-d_{\Sigma} \alpha \wedge H \wedge d t+\alpha \wedge J \wedge d t\right) \\
& =\int_{X} \alpha \wedge\left(d_{t} D-d_{\Sigma} H \wedge d t+J \wedge d t\right) .
\end{aligned}
$$

Setting this equal to zero by Hamilton's principle, one immediately gets Ampère's law as the sole Euler-Lagrange equation. The divergence constraint $d_{\Sigma} D=\rho$, corresponding to Gauss' law, has been eliminated via the restriction to the Weyl gauge. 
Noether's Theorem Implies Automatic Preservation of Gauss' Law. There are two ways that one can see why Gauss' law is automatically preserved, even though it has been eliminated from the Euler-Lagrange equations. The first is to take the "divergence" $d_{\Sigma}$ of Ampère's law, obtaining

$$
0=d_{\Sigma} d_{t} D-d_{\Sigma} d_{\Sigma} H \wedge d t+d_{\Sigma} J \wedge d t=d_{t}\left(d_{\Sigma} D-\rho\right) .
$$

Therefore, if this condition holds at the initial time, then it holds for all time.

A more "geometric" way to obtain this result is to use Noether's theorem, with respect to the remaining gauge symmetry $A \mapsto A+d_{\Sigma} f$ for scalar functions $f$ on $\Sigma$. To derive this, let us restrict $A$ to be an Euler-Lagrange solution in the Weyl gauge, but remove the previous requirement that variations $\alpha$ be fixed at the initial time $t_{0}$ and final time $t_{f}$. Then, varying the action along this new $\alpha$, the Euler-Lagrange term disappears, but we now pick up an additional boundary term due to integration by parts

$$
\mathbf{d} S[A] \cdot \alpha=\left.\int_{\Sigma} \alpha \wedge D\right|_{t_{0}} ^{t_{f}}
$$

If we vary along a gauge transformation $\alpha=d_{\Sigma} f$, then this becomes

$$
\mathbf{d} S[A] \cdot d_{\Sigma} f=\left.\int_{\Sigma} d_{\Sigma} f \wedge D\right|_{t_{0}} ^{t_{f}}=-\left.\int_{\Sigma} f \wedge d_{\Sigma} D\right|_{t_{0}} ^{t_{f}}
$$

Alternatively, plugging $\alpha=d_{\Sigma} f$ into $(\star)$, we get

$$
\mathbf{d} S[A] \cdot d_{\Sigma} f=\int_{X} d_{\Sigma} f \wedge J \wedge d t=-\int_{X} f \wedge d_{\Sigma} J \wedge d t=-\int_{X} f \wedge d_{t} \rho=-\left.\int_{\Sigma} f \wedge \rho\right|_{t_{0}} ^{t_{f}} .
$$

Since these two expressions are equal, and $f$ is an arbitrary function, it follows that

$$
\left.\left(d_{\Sigma} D-\rho\right)\right|_{t_{0}} ^{t_{f}}=0 .
$$

This indicates that $d_{\Sigma} D-\rho$ is a conserved quantity, a momentum map, so if Gauss' law holds at the initial time, then it holds for all subsequent times as well.

\section{GEOMETRIC DISCRETIZATION OF MAXWELL'S EQUATIONS}

Discretizing Maxwell's equations, while preserving the geometric properties mentioned above, can be achieved using cochains as discrete substitutes for differential forms, as previously done in, e.g., Bossavit [2]. Therefore, to compute Maxwell's equations, we begin by discretizing the 2form $F$ on a spacetime mesh $K: F$ assigns a real value to each oriented 2-face of the mesh. The exterior derivative $d$ is discretized by the coboundary operator, so the equation $d F=0$ states that $\left\langle d F, \sigma^{3}\right\rangle=\left\langle F, \partial \sigma^{3}\right\rangle=0$, where $\sigma^{3}$ is any oriented 3-cell in $K$ and $\partial \sigma^{3}$ is its 2-chain boundary.

Next, given a discrete Hodge star operator $[1,3,6,11], * F$ is a 2 -form on the dual mesh $* K$, while $\mathcal{J}$ is defined as a discrete dual 3 -form. Then, for every dual 3 -cochain $* \sigma^{1}$ (where $\sigma^{1}$ is the corresponding primal edge), the equation $d * F=\mathcal{J}$ becomes $\left\langle d * F, * \sigma^{1}\right\rangle=\left\langle * F, \partial * \sigma^{1}\right\rangle=\left\langle\mathcal{J}, * \sigma^{1}\right\rangle$.

When the cells $\sigma^{3}$ and $* \sigma^{1}$ are spacetimelike, then these correspond to the dynamical components of Maxwell's equations, and can be used to compute subsequent values of $F$. When the cells are purely spacelike, they correspond to the divergence constraint equations. The exact expression and update of these fields in time now depends on which type of mesh and time stepping method is desired, as described next.
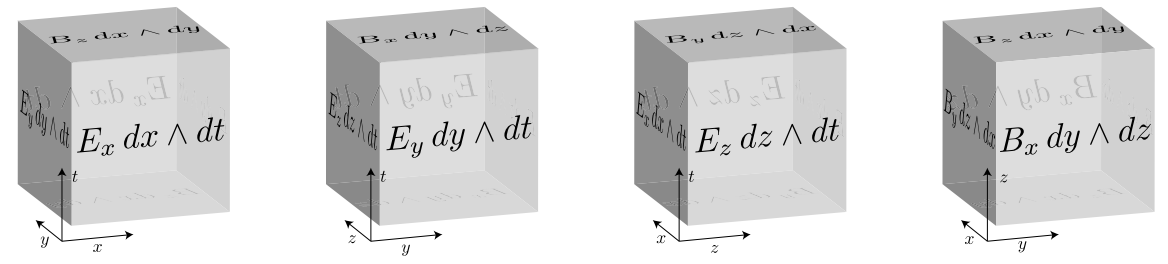

Figure 1: The 2-form $F=E \wedge d t+B$ can be discretized, on a rectangular spacetime mesh, by storing the components of $E$ and $B$ on 2D faces. The resulting numerical method is Yee's FDTD scheme. 
Uniform Time Stepping. For uniform rectangular meshes aligned with the $(x, y, z, t)$ axes, we can emulate the smooth coordinate expression of $F$ as

$$
\begin{aligned}
F= & E_{x} d x \wedge d t+E_{y} d y \wedge d t+E_{z} d z \wedge d t \\
& +B_{x} d y \wedge d z+B_{y} d z \wedge d x+B_{z} d x \wedge d y .
\end{aligned}
$$

This suggests the following discretization of $F$, shown in Figure 1: store $E_{x} \Delta x \Delta t$ on the $x t$-faces, $E_{y} \Delta y \Delta t$ on the $y t$-faces, and $E_{z} \Delta z \Delta t$ on the $z t$-faces; likewise for $B$. To discretize $\mathcal{J}$, we can similarly store $J_{x} \Delta y \Delta z \Delta t, J_{y} \Delta z \Delta x \Delta t, J_{z} \Delta x \Delta y \Delta t$, and $\rho \Delta x \Delta y \Delta z$ on the corresponding dual 3 -cells. If we then enforce the equations $d F=0$ and $d * F=\mathcal{J}$ in the discrete sense, the result is precisely the finite-difference time-domain (FDTD) integration scheme of Yee [12]. A similar procedure can be applied on unstructured (e.g., simplicial) spatial grids, on which we take uniform time steps $\Delta t$ (creating prism-shaped spacetime primal elements); in this case, solving the discrete Maxwell's equations recovers the more recent "Yee-like" method of Bossavit and Kettunen [4].

Asynchronous Time Stepping. As initially mentioned in Stern et al. [10], a more flexible integration scheme can be designed by assigning a different time step per element, in order to focus computational power where needed. A visualization of how to store $F$ on such a mesh structure is shown in Figure 2. This defines an asynchronous variational integrator that preserves the numerical properties of the uniform-stepping methods outlined above. The procedure to repeatedly update $E$ and $B$ asynchronously in time is as follows:

1. Select the face for which $B$ needs to be updated next.

2. $E$ advances $B$, using $d F=0$.

3. $B$ advances $E$ on neighboring edges, using $d * F=\mathcal{J}$.

Details of this algorithm, along with initial numerical results, can be found in Stern et al. [10], where only the case $\mathcal{J}=0$ was described.
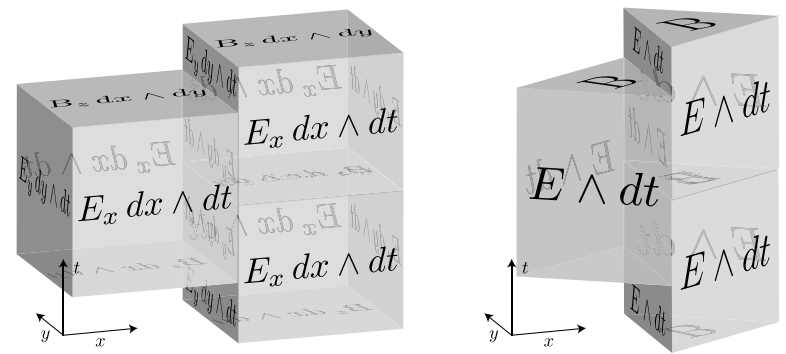

Figure 2: Here, $F$ is discretized on an asynchronous time-stepping grid, where each spatial element takes a different-sized time step from its neighbors. This can be done for either a rectangular spatial grid (left) or an unstructured/simplicial spatial mesh (right).

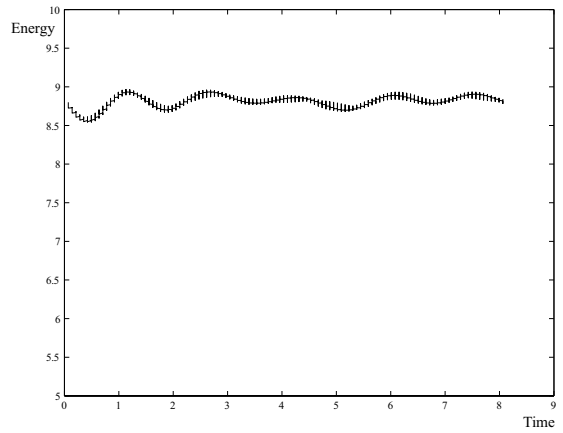

(a)

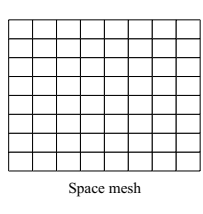

Figure 3: Our geometric integrator robustly maintains near-conservation of energy, even for asynchronous time stepping on a random spatial grid. (a) Regular grid, uniform time steps, (b) random grid, asynchronous time steps. 
One promising result from the initial numerical experiments, shown in Figure 3, is that this asynchronous integrator matches the FDTD scheme's excellent energy conservation behavior, even on a highly irregular grid, without exhibiting artificial damping or forcing. Future work is expected to include formal accuracy and stability analysis of this asynchronous integrator, focusing both on the choice of Hodge star and on resonance stability criteria for selecting the individual time steps.

\section{ACKNOWLEDGMENT}

Our research was partially supported by a Betty and Gordon Moore fellowship at Caltech, NSF grants CCR-0133983 and DMS-0453145 and DOE contract DE-FG02-04ER25657, and by NSF grant CCF-0528101. We gratefully acknowledge these sponsors for their support of this work.

\section{REFERENCES}

1. Auchmann, B. and S. Kurz, "A geometrically defined discrete Hodge operator on simplicial cells," IEEE Trans. Magn., Vol. 42, No. 4, 643-646, 2006.

2. Bossavit, A., Computational electromagnetism, Electromagnetism, Academic Press Inc., Variational formulations, complementarity, edge elements, San Diego, CA, 1998.

3. Bossavit, A. and L. Kettunen, "Yee-like schemes on a tetrahedral mesh, with diagonal lumping," Int. J. Numer. Modell., Vol. 12, Nos. 1-2, 129-142, 1999.

4. Bossavit, A. and L. Kettunen, "Yee-like schemes on staggered cellular grids: A synthesis between FIT and FEM approaches," IEEE Trans. Magn., Vol. 36, No. 4, 861-867, 2000.

5. Gross, P. W. and P. R. Kotiuga, "Electromagnetic theory and computation: A topological approach," Mathematical Sciences Research Institute Publications, Vol. 48, Cambridge University Press, Cambridge, 2004.

6. Hiptmair, R., "Discrete Hodge operators," Numer. Math., Vol. 90, No. 2, 265-289, 2001.

7. Lew, A., J. E. Marsden, M. Ortiz, and M. West, "Variational time integrators," Internat. J. Numer. Methods Engrg., Vol. 60, No. 1, 153-212, 2004.

8. Marsden, J. E. and M. West, "Discrete mechanics and variational integrators," Acta Numer., Vol. 10, 357-514, 2001.

9. Nédélec, J. C., "Mixed finite elements in $R^{3}$," Numer. Math., Vol. 35, No. 3, 315-341, 1980.

10. Stern, A., Y. Tong, M. Desbrun, and J. E. Marsden, "Computational electromagnetism with variational integrators and discrete differential forms," preprint at arXiv: 0707.4470 [math.NA], 2007.

11. Tarhasaari, T., L. Kettunen, and A. Bossavit, "Some realizations of a discrete Hodge operator: A reinterpretation of finite element techniques," IEEE Trans. Magn., Vol. 35, No. 3, 1494-1497, 1999.

12. Yee, K. S., "Numerical solution of inital boundary value problems involving Maxwell's equations in isotropic media," IEEE Trans. Ant. Prop., Vol. 14, No. 3, 302-307, 1966. 